\title{
Evaluación micológica en bosques de roble en el paisaje terrestre protegido "Miraflor-Moropotente" del municipio de Estelí- Nicaragua
}

\author{
Juan Carlos Vargas Fuentes ${ }^{1}$ \\ Josep Gribal ${ }^{2}$
}

\section{RESUMEN}

El presente estudio analiza los patrones de diversidad y distribución de macrohongos en el área protegida de Miraflor - Moropotente de Estelí-Nicaragua; teniendo como principales variables la altura y el estado de conservación de los bosques de roble de la zona (Quercus segoviensis y Quercus sapotaefolia), esto a fin de relacionar si las variables descritas inciden en la presencia o no de macrohongos, se designaron un total de 12 áreas de muestreos y se aplicaron diferentes índices de diversidad como son el índice de Shannon, Simpson y Sorensen para valorar la diversidad a niveles alfa y beta. Se encontraron 31 familias que agrupan 55 géneros con 85 morfoespecies; que significaron un total de 1722 individuos distribuidos en las diferentes áreas de muestreo. Podemos decir que en base a los resultados no hay diferencias significativas en cuanto a la diversidad de macrohongos tomando en cuenta las variables antes descritas.

Palabras Claves: Macrohongos, diversidad, distribución, índices de biodiversidad, conservación.

Recibido: 28 de agosto de 2015

Aceptado: 2 de octubre de 2015

1 Departamento de Ciencias tecnología y salud, Facultad Regional Multidisciplinaria de Estelí, UNAN-Managua. Correo Electrónico: jcarlos_vf@yahoo.es

2 Universidad Autónoma de Barcelona, España. Correo Electrónico: Josep.Gribal@uab.cat 


\title{
Mycological Evaluation in oak forests in protected terrestrial landscape "Miraflor - Moropotente" the municipality of Estelí - Nicaragua
}

\begin{abstract}
This study analyzes the patterns of diversity and distribution of macrofungi in the protected area of Miraflor - Moropotente, Esteli - Nicaragua, having as main variables the height and condition of the oak forests of the area (Quercus segoviensis and Quercus sapotaefolia), that relate to whether the described variables affect the presence or absence of macrofungi, were designated a total of 12 sampling areas and applied different diversity indices such as Shannon index, Simpson and Sorensen to value diversity alpha and beta levels. He found 31 families 55 genera grouped with 85 morphospecies, which meant a total of 1722 specimens distributed in the different sampling areas. We can say that based on the results that there is no significant difference in the diversity of macrofungi taking into account the variables described above.
\end{abstract}

Keywords: Macrofungi, diversity, distribution, rates of biodiversity, conservation. 


\section{INTRODUCCIÓN}

Los hongos son un grupo diverso de organismos unicelulares o pluricelulares que se alimentan mediante la absorción directa de nutrientes presentes en su sustrato. Junto con las bacterias, los hongos son los causantes de la putrefacción y descomposición de toda la materia orgánica. Se desarrollan en climas ecuatoriales, sub-tropicales o tropicales, templados y aún en los fríos; y desde el nivel del mar, hasta altitudes de 4,000 msnm (Herrera \& Ulloa, 1990). Son un grupo muy diverso de individuos con un papel ecológico importante, contribuyen a la formación de suelo y al reciclaje de elementos en los ecosistemas. Por su tipo de nutrición, tienen la capacidad de desdoblar materiales orgánicos tan complejos como lignina, celulosa y quitina (Guzmán, 1998).

El reino fungi se considera el segundo taxón más diverso después de los insectos y el menos estudiado, estimándose que se conoce solamente el $4.6 \%$ de la diversidad fúngica mundial. Las regiones tropicales y neo tropicales son las menos estudiadas en comparación con Europa y Norteamérica (Guzmán, 1998). En este sentido en Nicaragua, el tema de macrohongos está poco estudiado, según los datos de la recopilación de información de la biodiversidad Nacional no se reportan macrohongos (R. Pereira, 2007), esto limita directamente el conocimiento de su complejidad y riqueza, e impide la valoración de su estado en el territorio nacional, así como los usos y/o aprovechamientos que se podrían darles a estos.

El departamento de Estelí cuenta con 5 áreas protegidas, la seleccionada para la realización de este estudio fue la que se encuentra al noroeste del casco urbano de la ciudad de Estelí, conocida como "paisaje terrestre protegido Miraflor - Moropotente", la cual tiene una extensión total de 5,674 hectáreas (MARENA, 2006). Esta investigación consistió en identificar las especies de macrohongos de encontrados en bosques de roble Quercus segoviensis y Quercus sapotaefolia, en el área de Miraflor - Moropotente. Así mismo analizar la presencia o no de macrohongos en los bosques de roble en función de las condiciones del área (altitud y estado de conservación). Tomándose 3 alturas diferentes que rondaban entre los 1000 y 1300 msnm, y dos estados de conservación del bosque "poco conservado y conservado".

Como principales resultados encontramos que las áreas no presentan diferencias significativas en cuanto a la diversidad biológica al aplicar índices como el de Shannon, Simpson y Sorensen, no obstante podemos confirmar que los fenómenos biológicos en cuanto a la distribución espacial de especies de macrohongos en esta área es de un comportamiento normal, donde hay una separación perceptible en cuanto a la abundancia de las especies y la geometría de los remanentes cuando existe intervención antropogénica (Margalef, 1998).

\section{MATERIAL Y MÉTODOS}

Para la delimitación del área fue necesario tomar en cuenta ciertos criterios de selección: altura del lugar (se escogieron 3 zonas dentro del área núcleo de la reserva $(1000-1099$ msnm, 1100 - 1199msnm y 1200 - 1300msnm). También se escogió parcelas de muestreo donde el estado de conservación del bosque de roble tuviese dos criterios: poco conservado y muy conservado, de acuerdo a la densidad del remanente boscoso, por otra parte se escogieron sitios en donde el tipo de vegetación predominante correspondiera a las especies de (roble Montaña, "Quercus segoviensis y Quercus sapotaefolia”), y por último se trabajó exclusivamente en la zona núcleo del área protegida.

Los muestreos se realizaron en el período de invierno entre agosto y octubre del año 2012, para los que se aplicaron un total de 12 parcelas, 4 en cada altura con dos replicas por estado de conservación en los bosques de roble. Se procedió a realizar transectos de muestreo a conveniencia es decir que no tenían un rumbo definido, y estos fueron de aproximadamente 500 metros de longitud. Ver Mapa 1. 
Las personas que realizaron muestreos portaban guías para la identificación taxonómica en detalle de los hongos así como cintas para medir todas sus partes también se portaba con unas muestras de colores para el mismo objetivo.

Cada especie encontrada se recolectaba y se procedió a guardar en bolsas de papel encerado y posteriormente ubicarlas en una canasta, se marcó cada especie recolectada y se llevó control de la cantidad de especímenes que se encontraron por especie para determinar en los posteriores análisis la abundancia y dominancia por especie.

Todos los macrohongos que aparecieron dentro de cada transecto se fotografiaron con un mínimo de 3 fotografías en su estado natural y estrato correspondiente (suelo, hojarasca, madera, boñiga, raíces, entre otros). Posteriormente a este proceso cada especie se deshidrato para su almacenamiento y posterior estudio microscopico.

Para la identificación de las especies se tomaron el conjunto de los datos registrados en campo, se usaron claves para la identificación de los mismos (no en todos los casos), se viajó al Instituto Nacional de Biodiversidad de Costa Rica donde en conjunto Milagro Mata Hidalgo (especialista en biosistemática y taxonomía de hongos) que con guías taxonómicas y libros especializados ver referencias bibliográficas $(3$, $7,11,12,13,14,17)$ se lograron identificar la mayoría de macrohongos encontrados en las áreas de muestreo. En cuanto al análisis estadístico a niveles alfa fue necesario medir el índice de Diversidad de Shannon Weiner, para poder interpretar la diversidad de las diferentes especies de macromicetos muestreadas en las dos zonas por separado, también se realizó un análisis de la Densidad Relativa (D.R) y la Frecuencia de Ocurrencia (F.O \%) de acuerdo a las familias y los diferentes géneros taxonómicos, así mismo se aplicó el índice de Simpson para valorar lo relacionado a la abundancia de las especies. Por otra parte se midió el índice de Sorensen para el análisis de comunidades y para comparar dos áreas mediante la presencia/ausencia de especies en cada una de ellas, esto en cuanto a los análisis a niveles beta.

\section{RESULTADOS}

Se encontraron 31 familias que agrupan 55 géneros con 85 morfoespecies ${ }^{1}$; que significaron un total de 1722 individuos distribuidos en las diferentes áreas de muestreo ver Tabla 1.

\section{Tabla 1: Consolidado total de muestreos}

\begin{tabular}{llccc}
\hline $\mathbf{N}^{\circ}$ & \multicolumn{1}{c}{ Familias } & Géneros & Sp. & Individ. \\
\hline 1 & Agaricaceae & 7 & 7 & 141 \\
2 & Amanitaceae & 1 & 1 & 31 \\
3 & Auriculariaceae & 2 & 3 & 47 \\
4 & Bankeraceae & 1 & 1 & 9 \\
5 & Boletaceae & 7 & 13 & 166 \\
6 & Cantharellaceae & 1 & 1 & 78 \\
7 & Clavicipitaceae & 1 & 1 & 3 \\
8 & Cortinariaceae & 1 & 4 & 50 \\
9 & Entolomataceae & 1 & 1 & 30 \\
10 & Gyroporaceae & 1 & 1 & 26 \\
11 & Hygrophoraceae & 1 & 1 & 4 \\
12 & Hymenochaetales & 1 & 1 & 10 \\
13 & Inocybaceae & 1 & 1 & 20 \\
14 & Marasmiaceae & 5 & 12 & 420 \\
15 & Meruliaceae & 2 & 2 & 35 \\
16 & Mycenaceae & 1 & 4 & 22 \\
17 & Physalacriaceae & 1 & 1 & 39 \\
18 & Pluteaceae & 1 & 1 & 9 \\
19 & Polyporaceae & 2 & 3 & 13 \\
20 & Pterulaceae & 1 & 1 & 42 \\
21 & Ramariaceae & 1 & 1 & 15
\end{tabular}

1 Morfoespecie es un taxa rápidamente separable por diferencias morfológicas que son obvias para individuos sin extensivo crecimiento taxonómico, en un ordemamiento de morfoespecies es muy conveniente la determinación a alguna categoría formal como familia o preferiblemente género (Oliver y Beattie, 1995), posterior a esto las morfoespecies se pueden ordenar de la siguiente manera: Género X morfoespecie 1, por ejemplo Marasmius sp. 1 (Lattke, 2000) 


\begin{tabular}{llccc}
22 & Russulaceae & 2 & 7 & 72 \\
23 & Schizophyllaceae & 1 & 1 & 34 \\
24 & Schizoporaceae & 1 & 1 & 115 \\
25 & Sclerodermataceae & 1 & 1 & 28 \\
26 & Serpulaceae & 1 & 1 & 15 \\
27 & Steraceae & 2 & 2 & 147 \\
28 & Strophariaceae & 2 & 3 & 40 \\
29 & Trichocomaceae & 1 & 1 & 31 \\
30 & Tricholomataceae & 3 & 6 & 29 \\
31 & Xylariaceae & 1 & 1 & 1 \\
& & $\mathbf{5 5}$ & $\mathbf{8 5}$ & $\mathbf{1 7 2 2}$ \\
\hline
\end{tabular}

Del total de morfoespecies encontrados en los muestreos se ubican 24 familias en las áreas conservadas con un total de 1044 individuos y se encontraron 27 familias en el bosque poco conservado, con una abundancia de 678 ver Gráfico 1.

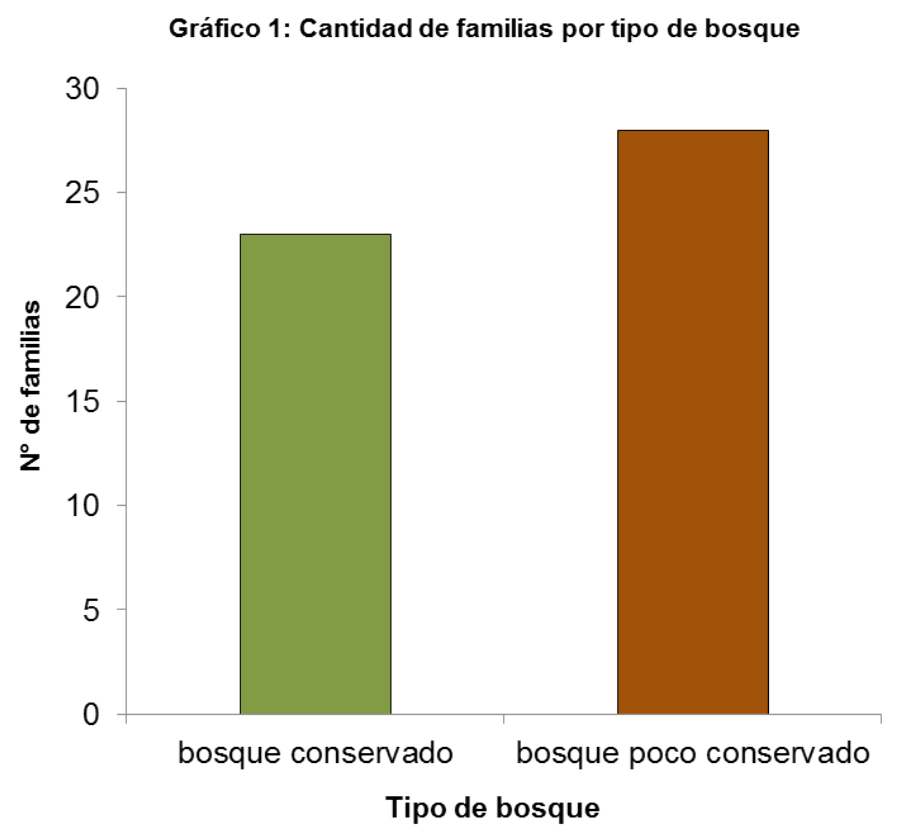

En cuanto a la cantidad de familias e individuos encontrados por tipo de bosque y altura apreciamos entre los 1000 - 1099 en bosque conservado un total de 21 familias con 360 individuos, en bosque no conservado 19 familias con 271 individuos, entre los 1100 - 1199 en bosque conservado 20 familias con 276 individuos; en bosque poco conservado 17 familias con 132 individuos y en las alturas entre $1200-1300$ encontramos en bosque conservado 23 familias con 528 individuos; en bosque poco conservado 20 familias y 275 individuos ver Gráfico 2 y 3.

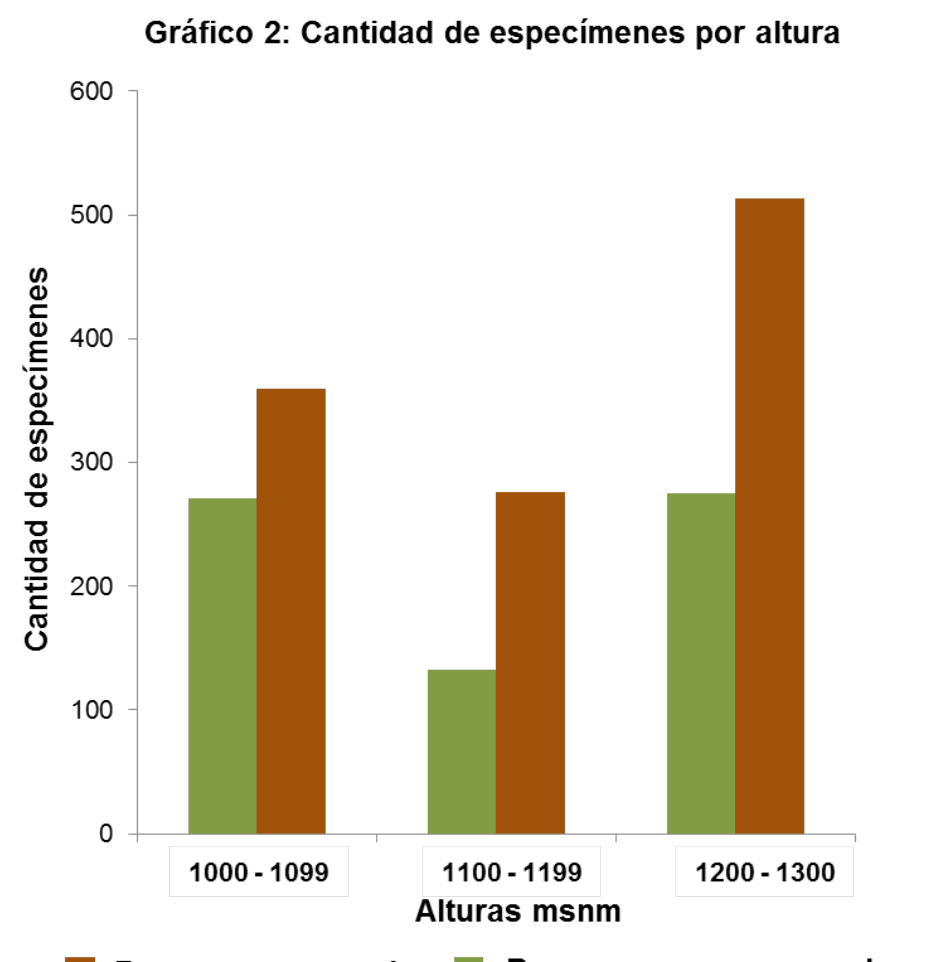

Bosque conservado

Bosque poco conservado

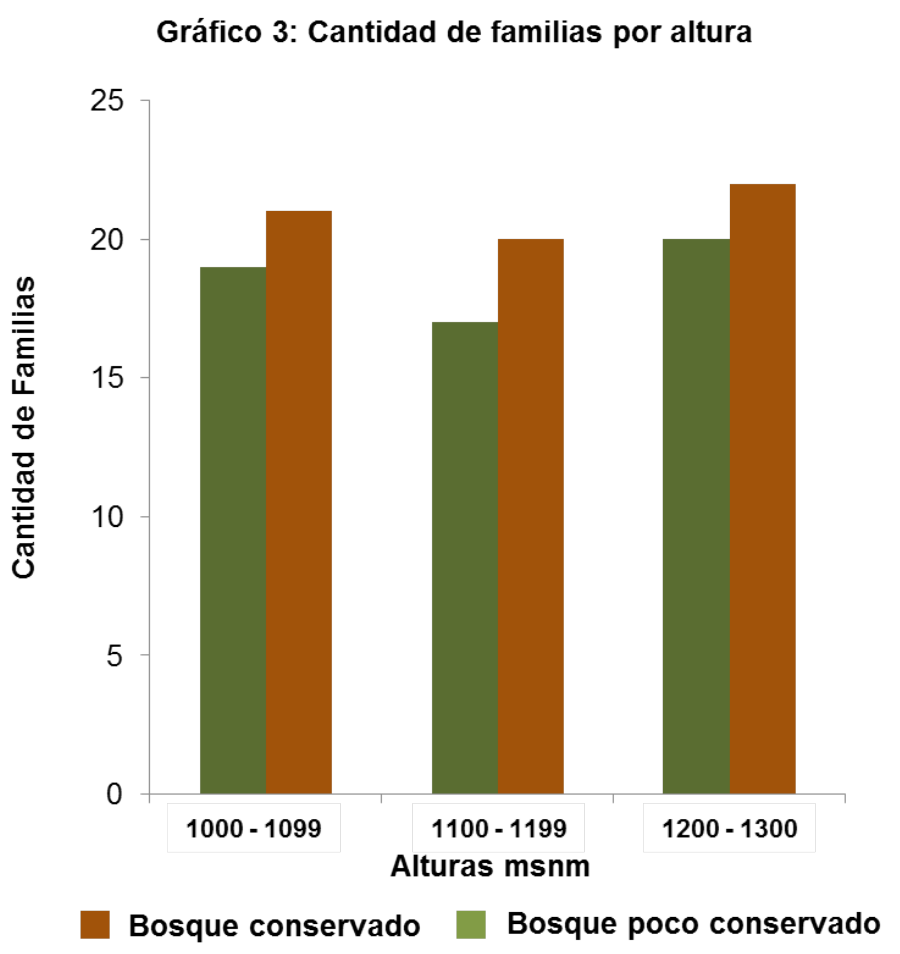


Dentro de las morfoespecies muestreadas las que mayor densidad relativa y frecuencia de ocurrencia obtuvieron están las especies que pertenecen a las familias; Marasmiaceae con una D.R dentro del bosque poco conservado del 20.1 y F.O de $19 \%$ para el caso del bosque conservado D.R: 28 y F.O: $27 \%$ y como segunda familia con mayor D.R y F.O esta la Boletaceae la cual en bosque conservado se encontró D.R: 6.7 y F.O $8 \%$ por otra parte en el bosque no conservado D.R: 14.3 y F.O: $15 \%$.

El índice de diversidad de Shannon para el bosque conservado fue de 2.622, para el caso del bosque poco conservado de 2.519. Si disgregamos la información por la variable altura encontraríamos que para el caso del bosque conservado en las alturas correspondientes a 1000 - 1099 msnm un índice de 2.298; de 1100 1199: 2.446 y entre 1200 - 1300: 2.494. Por otra parte en el caso del bosque poco conservado el índice de Shannon nos indicó que en alturas de 1000 - 1099 es de 2.348; entre 1100 - 1199: 2.356 y para la altura correspondiente a 1200 - 1300: 1.910 ver Tabla 2.

Tabla 2: Valores Índice de Shannon

\begin{tabular}{rcc}
\hline \multirow{2}{*}{ Altura } & \multicolumn{2}{c}{ Tipo de bosque } \\
\cline { 2 - 3 } & Conservado & Poco Conservado \\
\hline $1099-1100$ & 2.298 & 2.348 \\
$1199-1200$ & 2.446 & 2.356 \\
$1200-1300$ & 2.494 & 1.910 \\
Consolidado & 2.622 & 2.519 \\
\hline
\end{tabular}

Para el caso del índice de Simpson como principal resultado se obtuvo que en el bosque poco conservado el valor es de: 0.883 y en el conservado es de 0.890 .

En cuanto al índice de Sorensen se obtuvo como resultado de 0.80 de similitud entre ambos tipos de bosques, no obstante al aplicar el índice en ambos estratos boscosos por altura encontramos que entre 1000-1099: 0.80, de 1100-1199: 0.59 y entre 12001300: 0.67 de similitud.
Es importante resaltar que luego de realizar la identificación de macrohongos las especies Boletus cf. flavoruber y Serpula costaricensis ver Foto 1 y 2, son los primeros reportes realizados fuera de Costa Rica a nivel mundial (Mata, 2004).

\section{Foto 1}

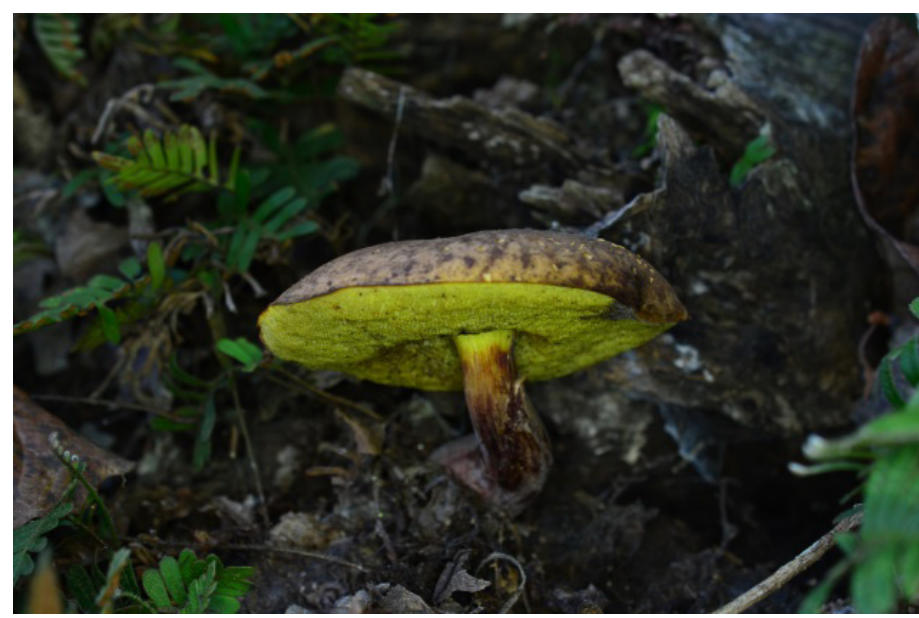

Fuente: Propia de la investigación

\section{Foto 1}

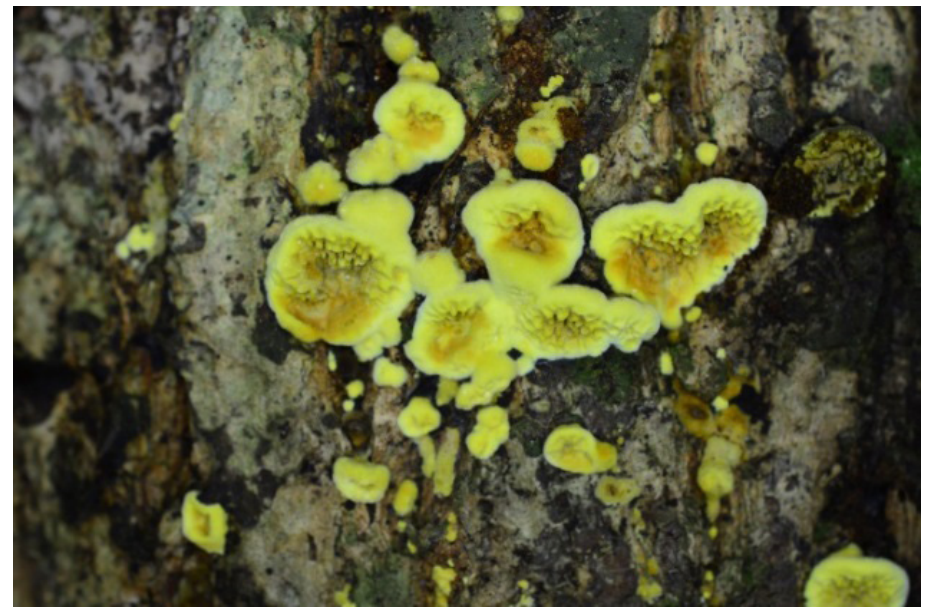

Fuente: Propia de la investigación

\section{DISCUSIÓN}

Ningún individuo, población o grupo de especies, se encuentra aislado ecológicamente, libre de las interacciones con otros organismos o poblaciones. Al contrario, todos los organismos están integrados 
en complejos ecológicos, con dinámicas complejas y ordenadas, que surgen de la relación entre componentes abióticos y bióticos. Para detectar estos patrones o relaciones es necesario conocer además de la diversidad, los factores que influyen (Mueller et al, 2004).

Los objetivos de este trabajo de investigación fueron orientados a conocer la diversidad y abundancia de las especies fúngicas de la zona estudiada, de esta manera se pudo comprobar que existe diversidad y abundancia en el Bosque "poco conservado" e igual en el bosque conservado, ya que las dos rutas muestreadas cuentan con condiciones ambientales, que favorecen a la fructificación de estas especies, pese a que las áreas son diferentes en cuanto a estado de conservación y altura, en ambas se observaron considerables cantidades de macromicetos.

Es importante mencionar que en ambas zonas se muestrearon especies del Subphylum Ascomycota y Basidiomycota; el Índice de Shannon-Wiener da el valor de $\mathrm{H}^{\prime}=2.622$ para el bosque conservado y $\mathrm{H}^{\prime}=$ 2.519 para el bosque poco conservado, estos valores solo demuestran que la diversidad es bastante similar en ambas áreas sin tomar en cuenta el número de carpóforos muestreados por otra parte este dato nos dice que las áreas son muy diversas.

Para el caso del índice del Simpson donde para ambos sitios el valor fue casi idéntico 0.883 y 0.890 , un número que se acerca a 1 , significa que en ambas áreas la probabilidad de un encuentro intraespecífico es muy poco probable ya que este índice asigna un gran valor las especies más abundantes. (Colwell, 2004), cabe destacar que este aspecto diferiría en las especies de las familias Marasmiaceas y Boletaceas puesto que para ambas áreas fueron las más abundantes.

Con respecto a la abundancia de macrohongos en remanentes de bosque con mejor conservación presentaron un mayor número de individuos (1029 individuos) ver Gráfico 2, y los remanentes en menor estado de conservación presentan una reducción notoria en el número de individuos colectados (678 individuos). (Bennet 1999), (Forman \& Godron 1986) apuntan que los remanentes con un mayor tamaño y conservación tienen mayor número de especies e individuos que las de menor tamaño. Los remanentes evaluados mantienen esta relación. En este sentido (Ávila, 2004), manifiesta que las plantas epífitas manifiestan un patrón similar, la riqueza y abundancia de morfoespecies y de epífitas disminuye con respecto a la perturbación de los sitios. Es importante destacar que la altura no incidió significativamente sobre la abundancia.

El índice de Sorensen nos indica que ambas áreas son símiles entre sí en cuanto a la diversidad de especies y que presentan en un $80 \%$ se similitud.

Por lo antes descrito se puede afirmar que tanto en las áreas de bosque de roble poco conservadas como las conservadas la diversidad es muy alta, puesto que los hongos como organismos descomponedores tienen una capacidad adaptativa sorprendente, al no ser organismos que dependan directamente de procesos fotosintéticos si no de la descomposición de materia orgánica (Mata, 2003), que en ambos bosque se encontraba presente.

Cabe mencionar la importancia de estos datos para esta Área Natural Protegida, pues se trata de un estudio pionero ya que hasta la fecha no se había realizado ningún estudio de esta naturaleza, los datos servirán para futuros estudios que profundicen en este tema y de los cuales se obtengan beneficios, pues algunas especies se sabe que son comestibles como Cantarellus cibarius y Lactarius indigo. Que podrían ser aprovechadas de manera controlada por medio de cultivos, por las poblaciones locales. 


\section{CONCLUSIONES}

Las zonas muestreadas del Bosque; conservado y poco conservado cuentan con abundancia y diversidad de especies fúngicas, específicamente macromicetos, puesto que cuentan con condiciones favorables para la aparición de especies fúngicas.

Los análisis de índices de biodiversidad de los macrohongos muestran que en los dos estratos boscosos (conservado y poco conservado) los cambios generados por el uso antropogénico del suelo; inciden significativamente en la abundancia de morfoespecies. Los macrohongos por su sensibilidad a los cambios en cobertura vegetal, pueden considerarse como indicadores potenciales de calidad del bosque para futuros monitoreos biológicos del área.

La altura no es un factor que limite directamente en la presencia de macrohongos en una determinada zona.

\section{REFERENCIAS BIBLIOGRÁFICAS}

Aldana , F., \& Halling, R. (2000). Setas de Colombia (Agaricales, Boletales, y otros Hongos) (Primera ed.). (U. d. Antioquía, Ed.) Colombia: Colciencias. Multimpresos.

Colwell, R. K., Mao, C., \& Chang , J. (2004). Interpolating, extrapolating and comparing incidence based species acumulation curves. Ecology.

Forman, R., \& Gordon, M. (1986). Landscape Ecology. United State: Jhon Wiley and Sons Icn.

Gerhardt, E., Vila, J., \& Limona , X. (2000). Hongos de España y Europa. Barcelona: Ediciones Omega S.A.

Guzmán, G. (1998). Analisis cualitativo y cuantitativo de la diversidad de los hongos en Mexico (Ensayo sobre el inventario fungico dle país) (La Diversdiad Biológica de Iberoamérica ed.). México.

Herrera, T., \& Ulloa , M. (1990). El Reino de los Hongos, Micología Básica y Aplicada (Primera ed.). México: UNAM \& Fondo de Cultura económica.

Indesxfundorum. (s.f.). Recuperado el 20 de Junio de 2013, de http://www.indexfungorum.org/names/ names.asp

Lattke, J. (2000). Specimen processing, Ants Standard methods for mesasure and monitoring biodiversity. Whashintong: Smithsoninan institute press.

MARENA. (2006). Informe nacional de áreas protegidas. Managua: Ministerio del ambiente y los recursos naturales .

Margalef, R. (1998). Ecologia. Barcelona: Omega Ediciones.

Mata, M. (2003). Macrohongos de Costa Rica. Costa Rica: Esitorial INBio.

Mata, M., Halling, R., \& Mueller, G. (2003). Macrohongos de costa Rica (Vol. II). Costa Rica: Editorial INBio.

Mueller, G., Bills, G., \& Foster, M. (2004). Biodiversity of Fungi, Invetory and Monitoring Methods. USA: Elsevier.

Oliver , I., \& Beattie, A. (s.f.). Invertebrate Morfospecies as Surrogates for species: A Case Study. Conservations Biology.

Pereira , R. (2007). Recopilación de la información sobre la biodiversidad de Nicaragua. Managua : Editorial INBio.

Pereira, R. (2007). Recopilación de la información sobre la biodiversidad de Nicaragua. Managua: INBio.

Toledo, J., \& Escobar, G. (1983). Hongos Salvadoreños . San Salvador: Editorial Universitaria S.A. 


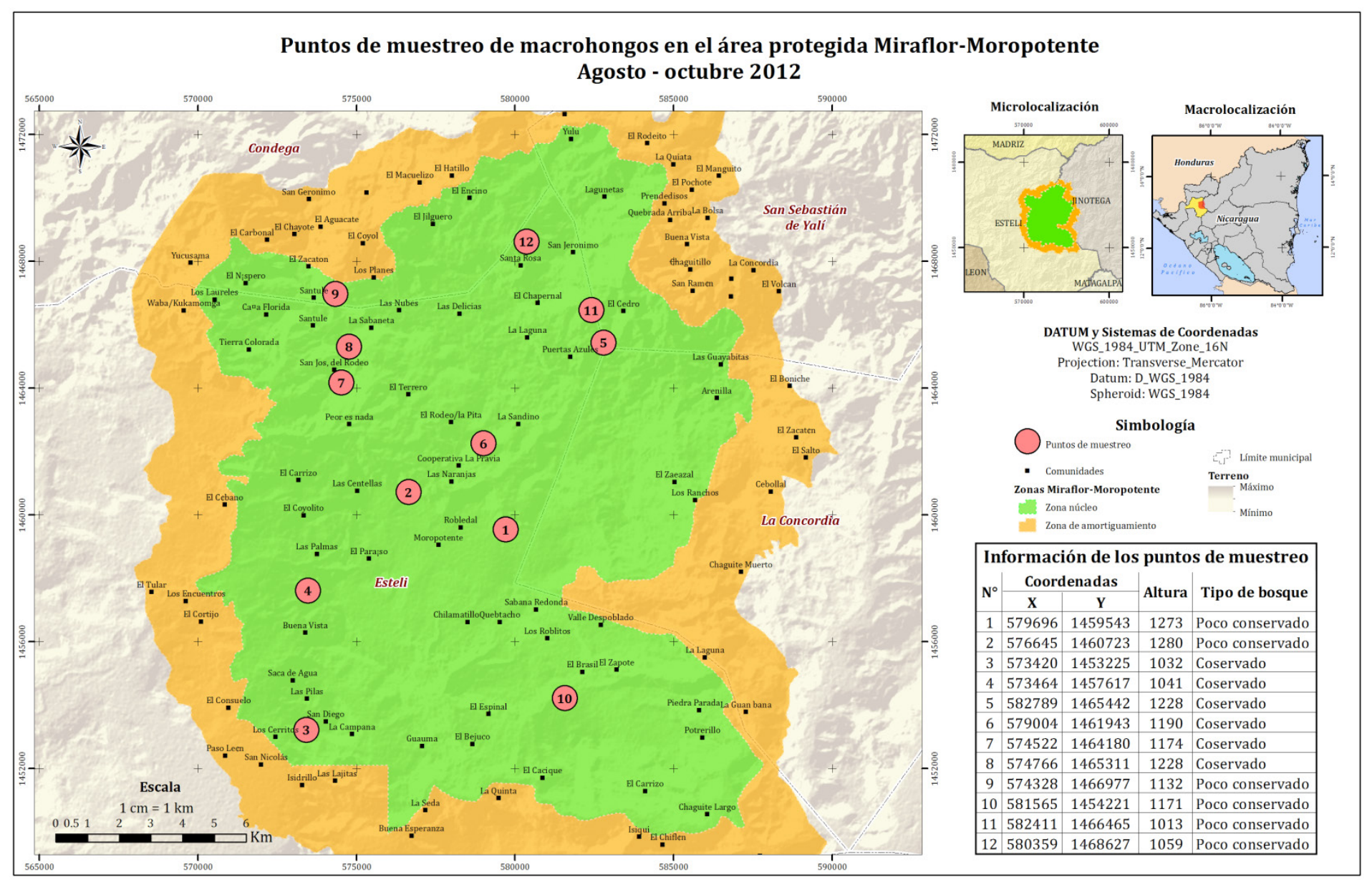

